\title{
Test validity defined as d-connection between target and measured attribute: Expanding the causal definition of Borsboom et al. (2004)
}

\author{
Peder M. Isager
}

2021-05-20

\section{Author note}

This article assumes some basic knowledge of causal graph model terminology. D-separation is a particularly relevant concept. For a brief but excellent introduction to d-separation, and the general framework of causal modeling, see Dablander (2020). For a more comprehensive introduction, see Pearl (2009) or Hernán \& Robins (2020). This article is written in response to Borsboom et al. (2004), and hence assumes some familiarity with the arguments put forward in that work.

\begin{abstract}
This article proposes a modification of the test validity definition put forward by Borsboom, Mellenbergh and van Heerden (2004). According to the original definition, a test is only valid if test outcomes are caused by variation in the target attribute. However, given that the goal of measurement is to derive a procedure for estimating true values of an unmeasured attribute, the requirement of causal influence of attribute upon measurement put forward by Borsboom et al. (2004) can be relaxed to a requirement of d-connection between the test and attribute. According to the d-connection definition of test validity, a test is valid for measuring an attribute if (a) the attribute exists, and (b) variation in the attribute is d-connected to variation in the measurement outcomes. In other words, a test is valid whenever test outcomes inform us either about what has happened to the target attribute in the past, or about what will happen to the target attribute in the future. Thus, the d-connection definition expands the number of scenarios in which a test can be considered valid. Defining test validity as d-connection between target and measured attribute situates the validity concept squarely within the structural causal modeling framework of Pearl (2009).
\end{abstract}




\section{Introduction}

Borsboom et al. (2009) give the following general definition of test/measurement validity: "Validity is a property of measurement instruments that [...] codes whether these instruments are sensitive to variation in a targeted attribute" (Borsboom et al., 2009, p. 135). A formalized definition is offered by Borsboom et al. (2004; henceforth BMH), who state that " $A$ test is valid for measuring an attribute if (a) the attribute exists and (b) variations in the attribute causally produce variation in the measurement outcomes" (BMH, p. 1061). The latter definition implies that a measure can only be valid when the attribute of interest is a cause of measurement outcomes. However, it is not clear from BMH why valid measurement should be restricted to this specific causal scenario.

This article argues that variations in an attribute need not causally produce variation in the measurement outcomes in order for the measurement instrument to be "sensitive to differences in the attribute". It is enough that the measure and the attribute are d-connected, which we can acknowledge by modifying the definition offered by BMH. What follows is an outline of such a modified definition, as well as a discussion of which simple causal scenarios can be considered valid measurement. For simplicity, the term measured attribute will be used as a general term for referring to a "test", a "scale", a "measurement tool", a "measurement instrument", or any other term referring to an observed variable that is used to estimate the values of a latent (or unmeasured) target attribute.

\section{D-connection definition of test validity}

Definition: A measured attribute is valid for measuring a target (unmeasured) attribute if (a) the target attribute exists and (b) if the target attribute is d-connected to (i.e. not d-separated from) the measured attribute, such that variation in the measured attribute is statistically associated with variation in the target attribute (for a definition of d-separation, see Pearl, 2009, definition 1.2.3; Hernán \&3 Robins, 2020, fine point 6.1). In other words, a measurement instrument is valid if, in the true causal graph, there is an "open path" between the instrument and the attribute that we want the instrument to measure.

D-connection between target attribute and measured attribute ensures that the instrument either gives us information about what has happened to the target attribute in the past, or about what is going to happen to the target attribute in the future. This proposed modification of BMH's definition has substantial implications for when a test would be considered valid for measuring an attribute. Importantly, validity is no longer restricted to situations where the measured attribute is caused by the target attribute. Valid measurement would also include cases where the target attribute is caused by the measured attribute, cases where measured and target attribute are not in a direct causal relationship but share a causal ancestor, and cases where measured and target attribute share a causal descendant that is conditioned on.

The rationale for preferring this d-connection definition over BMH's definition goes as follows: Criterion (b) in BMH's definition clearly implies that measurement involves gaining information about the values of one variable (the target attribute) by observing the values another variable (the measured attribute). Such information gain is possible when the target attribute causes the measured attribute. However, it is also possible in all cases where the target attribute is d-connected to the measured attribute. If there is no other justification for criterion (b) in BMH's definition, then we should relax this criterion to allow as valid all causal scenarios where observation of the measured attribute yields information about the target attribute.

The following sections discuss six basic examples of valid and invalid measurement according to the dconnection definition of validity. After this follows a consideration of why one might prefer measured attributes that adheres to BHM's definition, even if d-connection is considered the fundamental criterion for measurement validity. Finally, example statements from BMH are discussed where the d-connection definition leads to radically different conclusions. 


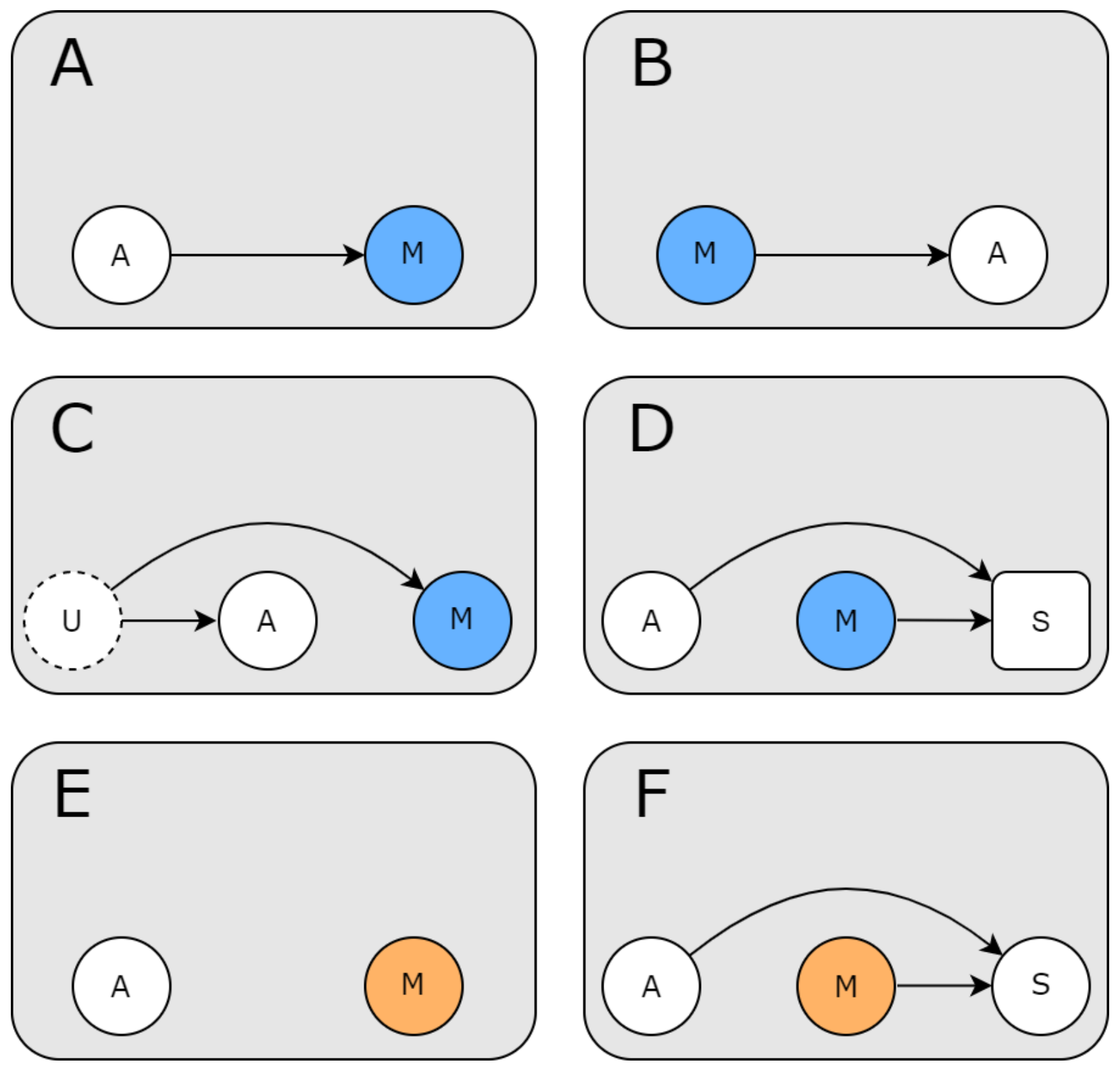

Figure 1: Causal models for various valid (1A-1D) and invalid $(1 \mathrm{E}-1 \mathrm{~F})$ measurement scenarios. $A=$ target attribute, $M=$ measured attribute, $U=$ unmeasured non-target attribute, $S=$ shared causal descendant. The square in panel D indicates that $S$ is being conditioned on. Blue coloring of $M$ in panel 1A to 1D indicates that $M$ is a valid measure of $A$. Orange coloring of $M$ in panel $1 \mathrm{E}$ and $1 \mathrm{~F}$ indicates that $M$ is an invalid measure of $A$. 


\section{Examples}

\section{Target attribute $\boldsymbol{A}$ causes measured attribute $M$}

Target attribute $A$ and measured attribute $M$ are d-connected when $A$ is a causal parent of $M$ (see figure 1A). In this case, variation in $M$ gives us information of what happened to $A$ in the past. This situation is what is covered in the definition given by BMH and is perhaps the most typical causal scenario for measurement instruments in many sciences. As an example, an MRI scanner can act as a measure of the spatial distribution of neural activity because it is sensitive to blood flow/oxygenation in various regions of the brain. It is a valid measure of neural activity in regions of the brain because neural activity $A$ has a causal effect on blood flow $M$ in the same region.

\section{Measured attribute $M$ causes target attribute $A$}

$A$ and $M$ are also d-connected when $M$ is a causal parent of $A$ (see figure 1B). In this scenario, variation in $M$ gives us information about what will happen to $A$ in the future. As an example, consider a company that wants to hire applicants that will perform well in their job. The company does not know what the applicants' future job performance $A$ will be, but they do have access to the applicants' past work experience $M$. If past work experience has a causal effect on future job performance, then $M$ is a valid measure of $A$ in this case.

Note that the example above would not be considered a case of valid measurement according to BMH's definition of validity, since variation in the target attribute "future job performance" does not causally produce variation in the measured attribute "past work experience". We return to this discrepancy between the d-connection definition and BMH's definition in the discussion.

\section{Target attribute $A$ and measured attribute $M$ are both caused by some third attribute $U$}

$M$ can be a valid measure of $A$ even when there is no direct causal relationship between $M$ and $A$. One example of this is when $M$ and $A$ share a causal parent $U$ (see figure 1C). In this case, $M$ gives us information about what happened to $U$ in the past. Information about what happened to $U$ in turn gives us information about what will happen to $A$ in the future, since $U$ causes $A$. As an example, consider again the company that wants to use past work experience $M$ to predict future job performance $A$. However, in this scenario there is no direct relationship between past work experience and future job performance. Both are simply caused by the applicants' education $U$. However, past work experience $M$ is still valid for measuring future job performance $A$ since past work experience $M$ is an indicator of the education $U$ an applicant has received, which in turn is an indicator of their future job performance $A$.

\section{Target attribute $A$ and measured attribute $M$ both cause some third attribute $S$ that is conditioned on}

Another example of when $M$ is valid for measuring $A$ - even when there is no direct causal relationship between them - is when $M$ and $A$ both have a causal influence on some shared descendant variable $S$, and we condition on $S$ before using $M$ to measure $A$ (see figure 1D). This is also known as conditioning in a collider (Rohrer, 2018). In causal modeling terms, the variable $S$ in figure 1 is called a collider variable (Pearl et al., 2016). Variables on the path on either side of a collider are d-separated from each other (Pearl, 2009, definition 1.2.3) unless they are conditioned on. When $S$ is conditioned on, the marginally blocked path becomes unblocked (Pearl et al., 2016). In general terms, we use the combined knowledge of the values of $M$ and $S$ to reason back to what $A$ must likely have been.

As an example, suppose we intend to use a person's height $M$ as a measure of their hand-eye coordination skills $A$. In the general population these attributes are unrelated. However, suppose we restrict our measure to professional basketball players in the Women's National Basketball Association (WNBA). In this case, we know that both good hand-eye coordination and being tall are important for performing well enough in basketball to play in the WNBA. Thus, WNBA players either have good coordination skills, are tall, or both. Women who are neither tall nor particularly sleight of hand will very likely not make it into the 
WNBA. Thus, if we observe a shorter-than-average WNBA player we can infer that they must likely have better-than-average hand-eye coordination to make up for their height disadvantage. Otherwise, they would not have made it into the WNBA. Consequently, height $M$ is a valid measure of hand-eye coordination $A$ conditional on being a WNBA player $S$ (assuming the causal relationships described above are true).

Valid measurement is not restricted to the examples above. Any causal scenario in which $A$ and $M$ are d-connected (assuming $A$ exists) represents a scenario where $M$ is a valid measure of $A$.

\section{Invalid measurement}

Invalid measurement occurs whenever the target attribute $A$ does not exist, since a measured attribute $M$ cannot be d-connected to a non-existing target attribute. Invalid measurement also occurs in situations where $A$ exists, but $A$ and $M$ are d-separated. This happens, for example, when $M$ and $A$ have no direct causal relationship and do not share any causal ancestors or descendants (figure 1E), and when $A$ and $M$ share a descendant which is not conditioned on (figure $1 \mathrm{~F}$ ).

Suppose again that we are using height to measure hand-eye coordination, but we are not conditioning on any common outcome $S$. In that case, $M$ would contain no information about $A$ and would not be a valid measure of $A$. In other words, whether a measurement instrument is valid depends not only on properties of $A$ and $M$, but also on properties of all variables that play a part in the d-connection between $A$ and $M$. This holds even if we assume BMH's more restrictive definition of test validity, since we can obviously alter the context of measurement to manipulate whether variation in target attribute $A$ will causally produce variation in measured attribute $M$ (e.g. by conditioning on mediating variables).

\section{Measuring target attribute scores vs. measuring the effect of treatment on the target attribute}

Although the d-connection definition of validity allows several causal scenarios to be considered valid measurement, there may be good reasons to prefer measurement tools where $A$ causes $M$.

One reason is that the validity of a test depends on whether the goal of the test is to predict target attribute values, or to detect change in the target attribute as a function of some treatment. In all scenarios in figure 1, we treat measurement as synonymous with prediction. The goal is to observationally estimate (or "measure", or "predict", or "gain accurate information about") the true values of $A$. Given that the causal model is true in each case, $M$ will be valid for estimating $A$ in figure 1A-D.

However, suppose we are conducting a randomized experiment where we want to measure the causal effect of some treatment $T$ on attribute $A$. Our target attribute of interest is no longer $A$ per se. Rather, we want to measure change in $A$ with respect to changes in $T$. In technical terms, we want the form of the function $f(T)$ in the structural equation that determines $A, A=f(T, U)$, where $U$ is all unmeasured causes of $A$ that are either experimentally controlled or randomized between groups in our experiment. For example, in a linear model we would want to know the value of the direct effect of $T, \beta T$, in the linear structural equation for $A$, $A=\beta T+\epsilon$.

Figure 2 displays the same causal models for the relationship between $A$ and $M$ as figure 1A and 1B (see figure $2 \mathrm{~A}$ and $2 \mathrm{~B}$ respectively), but now shows what happens if our target attribute is changed to $f(T)$. In figure $2 \mathrm{~A}$ our measured attribute $M$ is still valid since it remains d-connected with $f(T)$. However, in figure $2 \mathrm{~B}$, even though $M$ is d-connected to $A$, it is no longer d-connected to the target attribute $f(T)$ and ceases to be a valid measure. As an example, imagine an employer who wants to know the effect of a training program $T$ on future job performance $A$. The employer has access to information about employee's past work experience $M$, and she knows that work experience is a cause of future work experience. In that case, past work experience $M$ is a valid measure of future job performance $A$. However, $M$ is not valid for measuring the effect of the training program $T$ on performance $f(T)$. Regardless of how efficient the training program $T$ is, it cannot change the applicant's past work experience $M$, so $M$ is completely insensitive to the effect of the training program $f(T)$, and consequently it is insensitive to changes in $A$ following change in $T$.

The advantage of a measurement instrument caused by the target attribute $(A \rightarrow M)$ is that $\mathrm{M}$ will be 

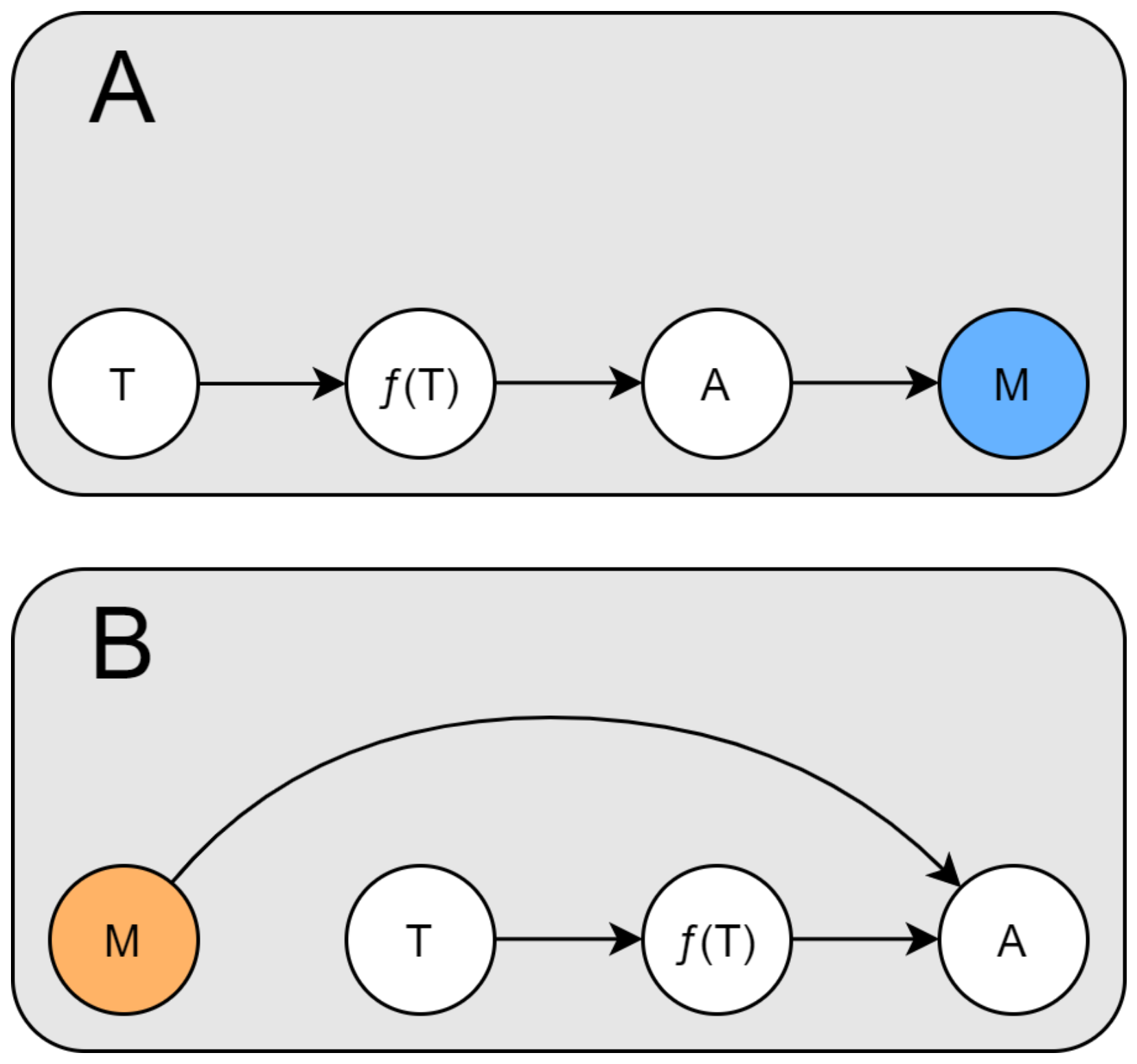

Figure 2: Measurement validity when the goal is to measure change in an attribute as a function of a given change in treatment. $A=$ attribute, $M=$ measured attribute, $T=$ treatment, $f(T)=$ change in attribute as a function of treatment. Blue coloring of $\mathrm{M}$ in panel $2 \mathrm{~A}$ indicates that $M$ is a valid measure of $A$. Orange coloring of $M$ in panel $2 \mathrm{~B}$ indicates that $M$ is an invalid measure of $A$. 
d-connected to both $A$ and any treatment effect $f(T)$ on $A$ (figure 2A). Thus, $M$ will remain valid regardless of whether our goal is simple prediction of $A$ or testing the effect of some treatment $T$ on $A$. We can always use the principle of d-connection to determine if $M$ is a valid measure. However, when $A$ is not a cause of $M$ we need to be very conscious about what target attribute we are interested in measuring.

\section{Using a measured attribute for conditioning on a target attribute that is a con- founder.}

Causal scenarios of the form $A \rightarrow M$ are also superior to other types of valid measurement when we want to estimate a causal effect $(X \rightarrow Y)$ and $A$ is a confounder which we need to condition on to accurately estimate the causal effect of $X$ on $Y$. In this case, a measured attribute $M$ can be used to (partially) block open paths through $A$, but only if $M$ is a causal descendant of $A$ (Greenland \& Pearl, 2014).

Figure 3 demonstrates the issue of using a measured attribute to condition on a target attribute in two different causal scenarios. Suppose we want to know if people who attend a job training program $X$ increase their job performance $Y$, and that this effect is confounded by job performance before training $A$. For example, people who already perform well at their job may be more likely to both attend the job training program, and to perform well at their job in the future. Suppose we cannot observe employee pre-training job performance $A$ directly. However, we have access to supervisor ratings of pre-training performance $M$ (figure $3 \mathrm{~A}$ ). If performance $A$ is a cause of ratings $M$ and we condition on $M$, we will at least partially condition on $A$ and block (some of) the back-door path between $X$ and $Y$. Contrast this with using past work experience $M$ as a measure of pre-training job performance $A$ (figure $3 \mathrm{~B}$ ). In this case we are no longer using a causal descendant of $A$ as the measured attribute. Thus, even if $M$ is valid for measuring $A$ according to the d-connection definition, $M$ cannot be used to condition on $A$ and block the back-door path between $X$ on $Y$. Whether this ability to condition on the target attribute should be a required property of a valid measurement instrument is left as an open question here. If one were to argue for this, one would then have to admit that the goal of measurement is no longer simply to construct instruments that "are sensitive to variation in a targeted attribute" (Borsboom et al., 2009).

\section{Points of similarity and divergence between BMH and the d- connection definition of validity}

In terms of philosophical foundations, the d-connection definition of test validity is identical to the definition of test validity offered by BMH. Validity is still considered to be a concept within the domain of ontology (as opposed to epistemology). Validity is still considered entity separate from reliability and measurement bias (however, zero reliability does imply no validity because a completely unreliable measure implies d-separation. I.e., variance in a completely unreliable measure cannot, by definition, be sensitive to variance in the attribute). Existence of the target attribute, and the causal relationship between target and measured attribute, are still the fundamental criteria used to determine validity. Finally, several issues raised by BMH are equally applicable to the d-connection definition, such as the distinction between intraindividual and interindividual measurement structures, and whether validity is best thought of as binary (true/false) or continuous entity.

The only difference between BMH's definition and the d-connection definition is whether validity holds only when the target attribute is a cause of the measured attribute, $(A \rightarrow M)$, or whether validity holds under any d-connected relationship between target- and measured attribute. Still, this one modification of the definition leads to very different conclusions about validity in several scenarios discussed by BMH.

As one example, consider the dissatisfaction of BMH with the statement by Guilford (1946) that "a test is valid for anything with which it correlates". BMH argue that "... the likelihood of encountering zero correlation in real life is exceedingly small, and especially in the social sciences, everything tends to correlate with everything (Meehl, 1978). Therefore, the upshot of any line of thinking that sees correlation as a defining feature of validity is that everything is, to some degree, valid for everything else. This absurdity does not arise in a causal theory because it is not the case that everything causes everything else." (BMH, p. 1066). In contrast, the d-connection definition of test validity is in practice closely aligned with the statement by 

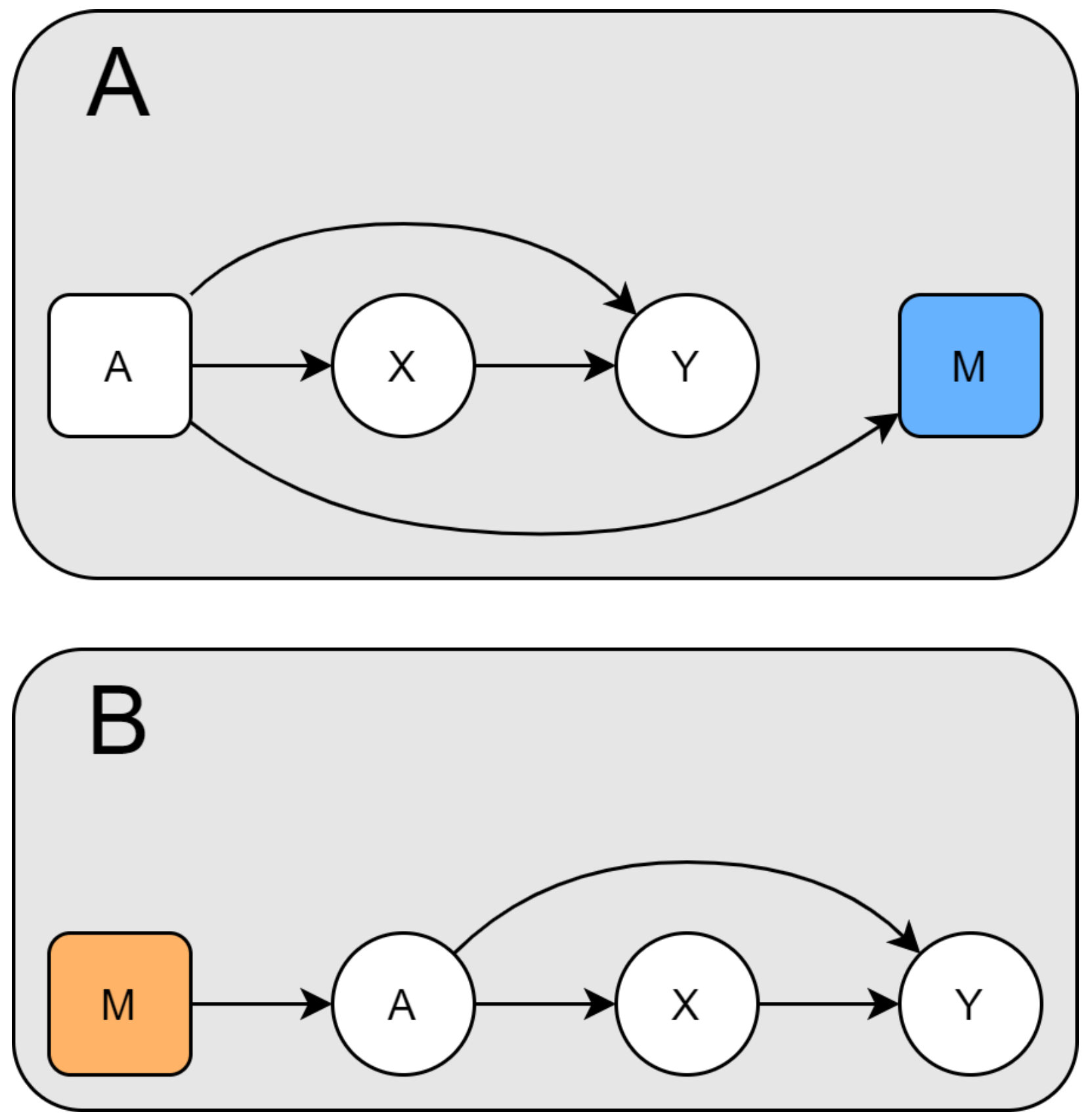

Figure 3: Measurement validity when the goal is to utilize the measurement for conditioning on a confounder. $A=$ confounding variable for the relationship $X \rightarrow Y, M=$ measured attribute, $X=$ cause, $Y=$ outcome. Square nodes represent those nodes that are (partially) conditioned on by conditioning on $M$. Blue coloring of $M$ in panel 3A indicates that $M$ is can (partially) block the confounding path between $X$ and $Y$ through $A$. Orange coloring of $M$ in panel 3B indicates that $M$ is not valid for blocking the confounding path through $A$. 
Guilford (1946). However, Guilford's statement does not suffice as a definition of test validity. A test "is valid for anything with which it correlates" only if the correlation is caused by d-connection between the test and the target attribute, and not by statistical noise or violation of causal identifiability conditions (Hernán $\&$ Robins, 2020). In addition, a test can be valid for measuring attributes with which the test does not correlate. One example of such a case is BMH's example of meter stick measurements of rods of equal length. Another is when the measure and attribute are strongly but non-linearly related (e.g., a measured attribute $M$ whose structural equation is the sine of $A$ ). In general, since validity is a statement about ontology (i.e., about causal processes in the real world), the language of causality must always be invoked to explain why correlation is (sometimes) an indication of valid measurement.

As another example of when the d-connection definition deviates from BMH, consider BMH's's statement that "Height and weight correlate about .80 in the general population, but this does not mean that the process of letting people stand on a scale and reading off their weight gives one valid measurements of their height". According to the d-connection definition, weight is a valid measure of height in this scenario, so long as height exists and is d-connected with weight. That is, so long as we can establish a d-connection relationship as the source of the correlation between height and weight, either will be a valid measure of the other. Some may object that we could easily imagine scenarios where a change in weight implies no change in height. If we run Mike Teavee through the taffy puller, he will become taller without gaining any weight. However, this is equivalent to imagining an intervention on height and wanting to know the effect of that intervention, which we can account for using the d-connection definition. If height $A$ and weight $M$ are both caused by common genes ( $U$; see figure 1C), then weight is not valid for measuring a treatment effect $f(T)$ on height. However, weight is still valid for measuring raw scores of height in the population. If you know how much a person weighs, you have (imperfect) information about how tall they are, even if some members of the population have been run through the taffy puller.

Finally, consider the statement made by $\mathrm{BMH}$ that in formative models "the observed indicators are not considered to be causally affected by the latent variable but, rather, to cause such a latent variable. In this case, it is difficult to see how these observed indicators could be conceptualized as measures of the attribute in question because the arrows between the attribute and the observations run in the opposite direction" (BMH, p. 1069). According to the d-connection definition of test validity, observed indicators are valid measures of target attributes in both latent variable models (figure 1A) and formative models (figure 1B). To appreciate why this makes sense, consider an extreme case where we know the exact structural equation that assigns values to a formative target attribute, and we have accurately measured all causal parents that enter into this equation. For example, many happily measure out 500 grams of sugar using a measuring cup, even though volume is a causal determinant of mass. We can do this because we know that mass equals volume times density, and we trust that the density of sugar is roughly accounted for by the scale printed on our measuring cup. In this case we can calculate values on our target attribute (weight of sugar in the cup) with near perfect certainty using only one measured cause of the attribute (volume of sugar in the cup) and an additional measurement assumption (sugar density is a constant that is corrected for). Surely this estimation procedure can still be considered a valid measure of the target attribute.

Note that in all the examples just mentioned, the d-connection definition is still in line with the more general definition of validity offered in Borsboom et al. (2009). That is, a measurement is "sensitive to differences in the attribute" as long as the measured and target attribute are d-connected.

\section{Implications of accepting the d-connection definition of validity}

Before accepting a d-connection definition of test validity, consider some of the stranger implications of the d-connection definition of test validity. The d-connection definition suggests that there is no substantive difference between terms such as "measure", "estimate", "predict", "determine", and "compute". It implies that we can measure what has not yet happened (unless one argues that what has not happened does not exist and, by extension, does not meet the requirement that the target attribute must exist). It also implies that a measurement instrument can be valid even if there is no direct causal path between the instrument and the attribute it purports to measure. 
The d-connection definition requires no particular causal proximity between measured and target attribute. The causal path from the target $A$ to the measured attribute $M$ may include any number of mediators (e.g. $A \rightarrow B \rightarrow C \rightarrow D \rightarrow E \rightarrow M$ ). Causal proximity is still important for measurement because a long mediating path implies many sources of error, which is important for measurement reliability. But causal proximity does not factor into whether a measurement is considered valid or not. In addition, the d-connection definition does not contain an explicit requirement of quantitative structure, and it is therefore somewhat detached from the classical conception of measurement utilized in the physical sciences (Michell, 2003).

The reader must critically evaluate whether these implications are acceptable. Based on my personal experience discussing these ideas with colleagues, I suspect many will be skeptical. This is good. The goal of this article is not to argue that the d-connection definition of validity represents the final word in the debate about what constitutes test validity. Rather, the goal is to add to that debate by highlighting what seems to be a conceptual issue in BMH's framework, and by proposing a modification (d-connection) that would seem to resolve this issue. Whether this modification is sensible or presents conceptual issues of its own remains a topic for discussion - the outcome of which will surely deepen our understanding of test validity even further.

\section{Acknowledgements}

I want to thank Stephen Antonopolis, Farid Anvari, Denny Borsboom, Fabian Dablander, Jaroslav Gottfried, Kai Horstmann, Daniël Lakens, André Nilsen, Julia Rohrer, Miguel Silan, Anna van 't Veer, and the Red^2 journal club, for helpful discussion and comments on earlier versions of this manuscript.

\section{References}

Borsboom, D., Cramer, A. O. J., Kievit, R. A., Scholten, A. Z., \& Franić, S. (2009). The end of construct validity. In The concept of validity: Revisions, new directions, and applications (pp. 135-170). IAP Information Age Publishing.

Borsboom, D., Mellenbergh, G. J., \& van Heerden, J. (2004). The Concept of Validity. Psychological Review, 111(4), 1061-1071. https://doi.org/10.1037/0033-295X.111.4.1061

Dablander, F. (2020). An Introduction to Causal Inference [Preprint]. PsyArXiv. https://doi.org/10.31234/o sf.io/b3fkw

Greenland, S., \& Pearl, J. (2014). Causal Diagrams. In N. Balakrishnan, T. Colton, B. Everitt, W. Piegorsch, F. Ruggeri, \& J. L. Teugels (Eds.), Wiley StatsRef: Statistics Reference Online (p. stat03732). John Wiley \& Sons, Ltd. https://doi.org/10.1002/9781118445112.stat03732

Guilford, J. P. (1946). New Standards For Test Evaluation. Educational and Psychological Measurement, 6(4), 427-438. https://doi.org/10.1177/001316444600600401

Hernán, M., \& Robins, J. (2020). Causal Inference: What If. Chapman \& Hall/CRC.

Meehl, P. E. (1978). Theoretical risks and tabular asterisks: Sir Karl, Sir Ronald, and the slow progress of soft psychology. Journal of Consulting and Clinical Psychology, 46(4), 806-834. https://doi.org/10.1037/0022006X.46.4.806

Michell, J. (2003). Epistemology of Measurement: The Relevance of its History for Quantification in the Social Sciences. Social Science Information, 42(4), 515-534. https://doi.org/10.1177/0539018403424004

Pearl, J. (2009). Causality: Models, reasoning, and inference. Cambridge University Press.

Pearl, J., Glymour, M., \& Jewell, N. P. (2016). Causal inference in statistics: A primer. John Wiley \& Sons Ltd.

Rohrer, J. M. (2018). Thinking Clearly About Correlations and Causation: Graphical Causal Models for Observational Data. Advances in Methods and Practices in Psychological Science, 1(1), 27-42. https://doi.org/10.1177/2515245917745629 